\title{
Vom Nutzen und Nachteil der Großwissenschaft. Altertumswissenschaftliche Unternehmungen an der Berliner Akademie und Universität im 19. Jahrhundert*
}

\author{
STEFAN REBENICH
}

»Der Malstrom hat mich zum erstenmal erfasst, als ich 1907 gleich nach dem Abitur in mein erstes Semester an die Universität Berlin eilte. Ich wollte keine Zeit verlieren, fleissig arbeiten und die weltberühmten Professoren hören.« Doch es dauerte nicht lange, bis die weltberühmten Professoren den katholischen Studiosus Carl Schmitt desillusioniert hatten. Die Vorstellungen, die der berühmte Philologe Ulrich von Wilamowitz-Moellendorff auf dem Katheder gab, empfand er als abstoßend; in dessen Goethe-Maske, die »drei bürgerliche Gesichter, das eines Predigers, eines Professors und eines Schauspielers« verband, sah er »das tiefste Unheil der Zeit«. »Die Mischung von preussischem Konservatismus und liberaler Fortschrittlichkeit, von Nationalismus und Humanismus«, von »betontem Selbstbewusstsein und ebenso betonter Bescheidenheit« war Schmitt rasch suspekt. Er flüchtete sich zu den Versen Friedrich Hölderlins. ${ }^{1}$

Etwa zur selben Zeit schrieb ein ungarischer Doktorand über seine Erlebnisse am Berliner Institut für Altertumskunde. Das Ergebnis war eine Satire: »Die Wissenschaft des Nicht Wissenswerten $\ll^{2}$ In einer Vorlesung über Catull erstickt der Ordinarius die herrlichsten Gedichte des jungen Dichters in einem Wust von textkritischen, überlieferungstechnischen und grammatikalischen Quisquilien. Die »Tatsachenknechtschaft des 19. Jahrhunderts« konnte der Autor nur mit Spott ertragen:

>Vivamus mea Lesbia atque amemus< [...]. Laß uns leben, meine Lesbia, und lieben, sagt der Herr im zerknitterten Rock auf dem Katheder, indem er seine bösen Äuglein

\footnotetext{
Der Vortragscharakter wurde im Wesentlichen beibehalten. Die Anmerkungen beschränken sich auf die wichtigsten Nachweise. Vgl. zum Thema Rebenich (1997); Rebenich (1999a) sowie Vom Bruch (2005) mit weiterführenden bibliographischen Angaben.

1 Tommissen (1990), 18 f.; vgl. Wirtz (2001), 415 .

2 Hatvany (1908/1986).
} 
auf zwei Bücher heftet. Das eine Buch ist seine Catullausgabe, das andere die eines Kollegen. ${ }^{3}$

Wie passen diese Momentaufnahmen zu dem Bild einer Altertumskunde, die sich um die Jahrhundertwende als Leitdisziplin wahrnahm, der nationale und internationale Beobachter Weltgeltung zubilligten und deren Publikationen und Unternehmungen als Maßstab moderner Wissenschaft galten? Was trieb die Forschenden an und was war das Ziel ihrer Forschungen? Welche Bedeutung hatten die Wissenschaften vom Altertum in der scientific community? Wo lagen ihre Chancen und wo lagen ihre Grenzen?

Ich will diese Fragen mit Blick auf die altertumswissenschaftlichen Unternehmungen der Berliner Akademie im 19. Jahrhundert zu beantworten suchen und werde mich dem Thema in fünf Schritten nähern. Zunächst gilt meine Aufmerksamkeit dem institutionellen Rahmen der Altertumsforschung. Hier wird die Organisation und Assoziation der Arbeit an dem Ort der altertumswissenschaftlichen »Großforschung « darzustellen sein: an der Preußischen Akademie der Wissenschaften. Sodann untersuche ich die wissenschaftspolitischen Bedingungen, die es ermöglichten, die großen akademischen Unternehmungen erfolgreich zu realisieren. In einem nächsten Schritt ist nach den theoretischen und epistemologischen Grundlagen dieser Big Science zu fragen, bevor die wissenschaftsimmanenten Folgen einer in Großprojekten sich organisierenden Altertumsforschung benannt werden. Resïmierend will ich Vor- und Nachteile dieser Forschung für die Wissenschaft vom Altertum bilanzieren und dieses Erbe aus heutiger Perspektive kritisch sichten.

\section{Die Altertumswissenschaften an der Preußischen Akademie der Wissenschaften}

Der Beginn der »Großwissenschaft «, der Beginn von Big Science oder Big Scholarship in der Antikenforschung lässt sich auf den Tag genau datieren: auf den 27. April 1858. Damals wurde Theodor Mommsen zum ordentlichen Mitglied der Berliner Akademie der Wissenschaften gewählt. ${ }^{4}$ In seiner Antrittsrede zeigte er am Beispiel des lange umstrittenen lateinischen Inschriftencorpus, welche Bedeutung der Akademie zukam. Wie in den naturwissenschaftlichen Disziplinen könne auch auf dem Gebiet der Altertumswissenschaft nur die straffe Organisation die »Archive der Vergangenheit« ordnen und damit die Voraussetzung schaffen, um zu neuen historischen Erkenntnissen zu gelangen. Hierzu sei es zum einen notwendig, sich der Unterstützung und Mitarbeit fähiger deutscher und

3 Hatvany (1908/1986), 6 und 24.

4 Zuvor war er bereits korrespondierendes Mitglied gewesen (seit dem 16. Juni 1853); vgl. Harnack (1900), Bd. 2, 189 sowie Kirsten (1985), Nr. 14, 81 f. 
ausländischer Wissenschaftler zu versichern. Zum anderen brauchten die großen Unternehmen bedeutende Geldmittel vom Staat. Schließlich liege es an seiner Generation, in der Akademie die unfruchtbare und traditionelle $»$ Arbeitszersplitterung « in der klassischen Altertumsforschung zu überwinden, indem Geschichte, Philologie und Jurisprudenz zusammenwirkten. ${ }^{5}$

In den folgenden Jahrzehnten seiner Mitgliedschaft in der Königlich Preußischen Akademie der Wissenschaften setzte Mommsen diesen >Arbeitsplan< konsequent und erfolgreich in die Tat um und prägte nachhaltig die Institution, deren Secretar er von 1874 bis 1895 war. ${ }^{6}$ Durch seine Initiative und unter seiner Führung entstanden die großen altertumswissenschaftlichen Unternehmungen, die quellenkritische Grundlagenforschung betrieben und die in hohem Maße dazu beitrugen, den internationalen Ruhm der deutschen Altertumswissenschaft zu begründen. Mommsen förderte systematisch die Expansion der akademischen Vorhaben und brachte seine Erfahrungen aus der Arbeit am Inschriftencorpus ein, um die Berliner Akademie zu einem »Großbetrieb der Wissenschaften « umzustrukturieren.

Nachdem 1874 der Haushalt der Akademie mehr als verdreifacht worden war, ging Mommsen in seiner Festrede vom 2. Juli auf die neue Situation ein. Es war seine erste Ansprache als Secretar der Akademie. Eindrucksvoll zeigte er am Beispiel seiner Disziplin, dass noch immer wichtige Vorarbeiten fehlten, die ein einzelner Forscher nicht leisten könne. Abhilfe schaffe einzig die Konzentration individueller Kräfte und die Organisation der Arbeit. Doch dies allein reiche nicht aus.

Alle die wissenschaftlichen Aufgaben, welche die Kräfte des einzelnen Mannes und der lebensfähigen Association übersteigen, vor allem die überall grundlegende Arbeit der Sammlung und Sichtung des wissenschaftlichen Apparates muss der Staat auf sich nehmen, wie sich der Reihe nach die Geldmittel und die geeigneten Personen und Gelegenheiten darbieten. Dazu bedarf es eines Vermittlers, und das rechte Organ des Staates für diese Vermittelung ist die Akademie. ${ }^{8}$

Mommsen, der Altertumswissenschaftler, hatte damit coram publico die Aufgabe der Akademie neu definiert und die Pflichten des Staates klar benannt. Zugleich unterstitzte er die Revision der Statuten, so dass die Berliner Akademie auch durch eine modernisierte Verfassung den neuen wissenschaftsorganisatorischen Erfordernissen gerecht werden konnte. ${ }^{9}$ Die Akademie hatte jetzt auch Stiftungen $\mathrm{zu}$ verwalten, die wissenschaftlichen Zwecken dienten, und trug somit der wach-

5 Mommsen (1858), wiederabgedruckt in ders. (1905), 35-38 (Zitate S. 37 und 35).

$6 \mathrm{Zu}$ Mommsens Rolle in der Berliner Akademie vgl. Hirschfeld (1904), wiederabgedruckt in ders. (1913), 931-965 sowie Rebenich (1999a).

7 Zum Begriff vgl. Harnack (1905), wiederabgedruckt in ders. (1911). Zum Hintergrund vgl. etwa Schiera (1992); Nowak (1996); Rebenich (2001b).

8 Mommsen (1874), zitiert nach ders. (1905), $46 \mathrm{f}$.

9 Vgl. Walther (1999). 
senden Bedeutung privater Mittel für die Finanzierung akademischer Unternehmungen Rechnung.

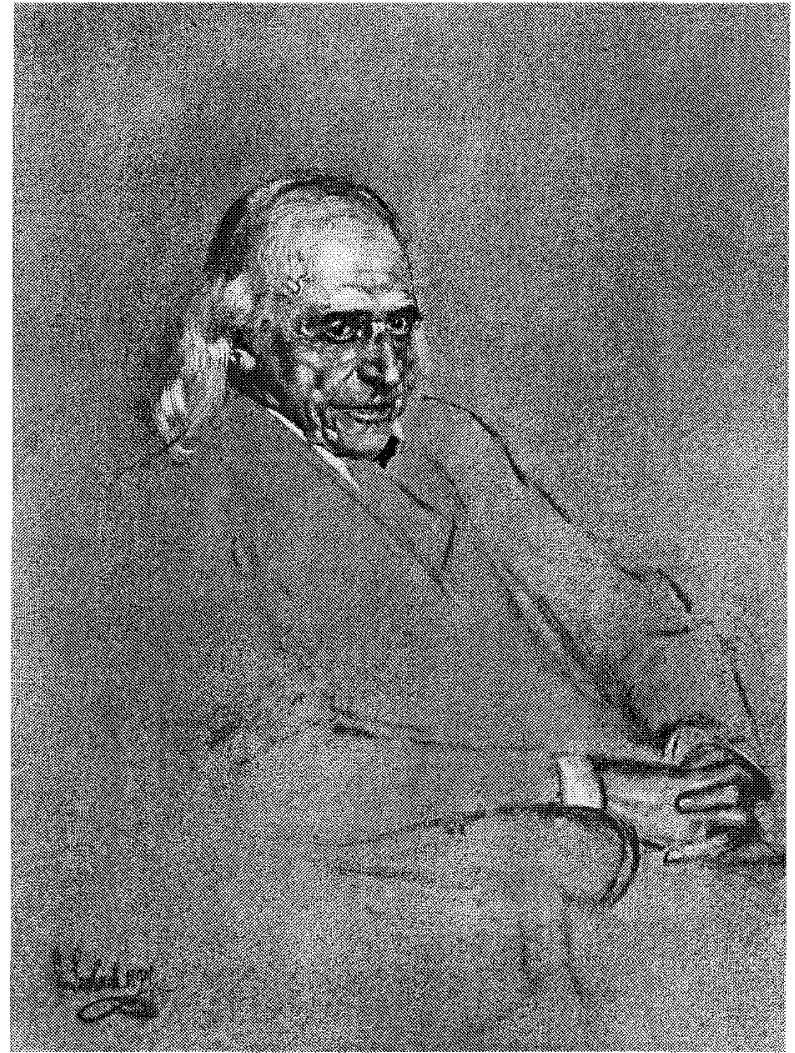

Abb. 1: Theodor Mommsen im Jahr 1897.

Die Preußische Akademie der Wissenschaften war für Mommsen weniger eine Stätte des gelehrten Diskurses, sondern eine Einrichtung, die die Voraussetzungen zur arbeitsteiligen Großforschung gewähren musste. Diese leistungsfähige Institution des Industriezeitalters hatte nichts mehr mit der traditionellen Honoratiorenvereinigung zu tun, die August Boeckh noch geschätzt hatte, der mit dem Corpus inscriptionum Graecarum das älteste wissenschaftliche Unternehmen der Akademie auf den Weg gebracht hatte. Zur Transformation der Akademie gehörte auch die forcierte Monopolisierung der Entscheidungen durch einzelne herausragende Akademiemitglieder, in der Regel die Secretare, die zugleich in zahlreichen Kommissionen und Stiftungskuratorien saßen und auch 
außerhalb der Akademie Funktionen und Ämter kumulierten. Dies ermöglichte ihnen, die akademische Forschungspolitik effizient zu kontrollieren. Mommsen etwa war neben seiner Tätigkeit in fast allen altertumswissenschaftlichen Kommissionen der Akademie zeitweise auch Mitglied der Savigny-Stiftung, die seit 1863 rechtshistorische Forschungen unterstützte, der Eduard Gerhard-Stiftung, die seit 1893 ein archäologisches Reisestipendium vergab, sowie der Charlottenstiftung, die 1874 von der Witwe Charlotte Stiepel, geb. Freiin von Hoffgarten, testamentarisch eingerichtet wurde und junge deutsche Philologen, die ihre Universitätsstudien vollendet, aber noch keine feste Anstellung gefunden hatten, auf die Dauer von vier Jahren förderte. Schließlich hatte er zeitweise den Vorsitz des Kuratoriums der 1894 eingerichteten Wentzel-Heckmann-Stiftung inne. Wilamowitz beklagte, Mommsens Beispiel vor Augen, den »Despotismus « der Direktoren, gegen den die einfachen Mitglieder der akademischen Kommissionen nur schwerlich etwas auszurichten vermochten. ${ }^{10}$ Arnaldo Momigliano wiederum sprach Jahrzehnte später mit Blick auf das Inschriftencorpus und die Monumenta Germaniae historica von der »tirannia di Mommsen«, fügte jedoch hinzu: »La tirannia di un Mommsen non sarebbe superflua oggi. « ${ }^{11}$

Die personelle Verschränkung zwischen Akademie und Universität war eng. Die altertumswissenschaftlichen Ordinarien der Friedrich-Wilhelms-Universität waren in der Regel Mitglieder der philosophisch-historischen Klasse der Akademie. Bei einzelnen Berufungsverhandlungen, wie etwa der Otto Hirschfelds, ${ }^{12}$ wurde zudem die Mitgliedschaft in Aussicht gestellt, um das Einkommen des zu Berufenden zu erhöhen. Da die materiellen Ressourcen der Akademie wesentlich größer waren als die der einzelnen Lehrstühle, war es nur folgerichtig, dass nicht die universitären Seminare die Träger des wissenschaftlichen Fortschritts auf dem Gebiet der griechisch-römischen Altertumskunde waren, sondern vielmehr die akademischen Großprojekte, die methodisch und inhaltich die Entwicklung der altertumswissenschaftlichen Disziplinen - keineswegs nur in Deutschland beeinflussten. Daran änderten auch die Schaffung neuer Lehrstühle und die Gründung altertumswissenschaftlicher Institute - wie etwa 1885 in Berlin ${ }^{13}$ - nichts. Das Berliner Institut für Altertumskunde sollte allerdings die alte $» V o r l e s u n g s-$ universität《 durch eine moderne »Arbeitsuniversität《 ersetzen helfen, die zumindest einen Teil der begabten Studenten an dem wissenschaftlichen Diskurs teilhaben ließ und in der akademischen Lehre die Vereinigung der einzelnen altertumswissenschaftlichen Fächer voranbringen sollte. Die eigentliche Forschung

10 Vgl. Brief Wilamowitz' an Eduard Schwartz vom 15. November 1901, zitiert nach Calder/ Fowler (1986), 31.

11 Momigliano (1979), 316.

$12 \mathrm{Vgl}$, dazu den umfangreichen Briefwechsel zwischen Theodor Mommsen und Friedrich Althoff, dessen Edition zur Zeit von Gisa Franke und Stefan Rebenich für die von der Historischen Kommission bei der Bayerischen Akademie der Wissenschaften herausgegebene Reihe Deutsche Geschichtsquellen des 19. und 20. Jahrhunderts vorbereitet wird.

13 Vgl. hierzu Unte (1985), 730-734. 
indes vollzog sich in außeruniversitären Einrichtungen wie der Akademie der Wissenschaften.

Hierzu fügt sich, dass die universitären Fachvertreter zumeist ihre wissenschaftliche Sozialisierung in den akademischen Kommissionen erfahren hatten; denn diese Kommissionen dienten gleichzeitig der Rekrutierung des wissenschaftlichen Nachwuchses, wie das Beispiel des Corpus inscriptionum Latinarum eindrucksvoll zeigt. Otto Hirschfeld, Hermann Dessau, Emil Hüibner, Elimar Klebs, Alfred von Domaszewski, Heinrich Dressel, Christian Hülsen, Johannes Schmidt, August Mau, Karl Zangemeister und Eugen Bormann zählten zur groBen Zahl der epigraphischen Mitarbeiter des Inschriftencorpus, die später mit Mommsens tatkräftiger Unterstiitzung in unterschiedlichen Sparten des Wissenschaftsbetriebs ihr Auskommen fanden.

In immer neuen Unternehmungen sollten alle erhaltenen Zeugnisse der römischen Antike gesammelt und ausgewertet werden. Die Berliner Akademie edierte griechische Inschriften, gab Aristoteles heraus und unterstützte die Veröffentlichung der byzantinischen Historiker. Nach Mommsens Eintritt 1858 vervielfältigte sich die Zahl der altertumskundlichen Projekte rasch. ${ }^{14} \mathrm{Zu}$ dem lateinischen Inschriftencorpus, das schon 1854 bewilligt worden war, trat 1874 die Prosopographie der römischen Kaiserzeit des ersten bis dritten Jahrhunderts. Ende der achtziger Jahre rief Mommsen die Kommission für Numismatik ins Leben, die mit der Sammlung der antiken Münzen Nordgriechenlands ihre Arbeit aufnahm. Darüber hinaus engagierte er sich für ein Wörterbuch der römischen Rechtssprache, eine neue Fronto-Ausgabe, eine umfassende Sammlung der Papyri, einen sachlich geordneten Katalog aller überlieferten Einzeldaten zum römischen Militärwesen der Kaiserzeit und die Herausgabe des Codex Theodosianus. Gemeinsam mit Adolf Harnack ging er daran, die Griechischen Christlichen Schriftsteller der ersten drei Jahrhunderte zu edieren und eine Prosopographie der Spätantike zu begründen. Kurzum: Die erfolgreichsten Unternehmen der editorischen und historischen Grundlagenforschung des Kaiserreichs erstreckten sich vor allem auf die Altertumswissenschaften.

Mommsens kategorische Forderung, das gesamte Quellenmaterial des römischen Altertums zu sichten und in umfassenden, kritischen Ausgaben vorzulegen, setzte nicht nur die Kooperation einzelner Wissenschaftler, sondern vielmehr ganzer Organisationen voraus. Es war offenkundig, dass die organisatorischen und die finanziellen Ressourcen der Preußischen Akademie nicht genügten, um alle Großunternehmen zu realisieren. Neue Strategien der Forschungsfinanzierung und Wissenschaftsorganisation mussten entwickelt werden, die in Zukunft für altertumswissenschaftliche Großprojekte richtungweisend waren und die sich andere Fächer zu eigen machten. Zum einen bot sich die Möglichkeit, die Reichsadministration zur Übernahme einzelner Institute oder langfristiger wissenschaft-

14 Vgl. Rebenich (1999a), 224-230 mit ausführlichen Literaturhinweisen. Zum Griechischen Münzwerk vgl. auch von Kaenel (2004). 
licher Unternehmen zu bewegen. Diese Konzeption wurde realisiert bei dem Archäologischen Institut und der Römisch-Germanischen Kommission, bei den Monumenta Germaniae historica, beim Deutschen Historischen Institut in Rom und bei der Reichslimeskommission. In den Direktorien dieser Einrichtungen saßen von der Preußischen Akademie gewählte Mitglieder. Zum anderen arbeiteten die deutschsprachigen Akademien der Wissenschaften zusammen, um große Vorhaben wie den Thesaurus linguae Latinae und die sechsbändige Enzyklopädie der mathematischen Wissenschaften (1895-1934) zu verwirklichen.

Mommsens Erbe in der Akademiepolitik trat Adolf Harnack an. Als der Kirchenhistoriker 1890 nicht aufgrund seiner theologischen, sondern vielmehr wegen seiner historischen Arbeiten in die Preußische Akademie aufgenommen wurde, begrüßte ihn Mommsen als denjenigen, der die Gabe besitze, »jüngere Genossen zu fruchtbarer Arbeitsgemeinschaft zu gewinnen und bei derjenigen Organisation, welcher die heutige Wissenschaft vor allem bedarf, als Führer aufzutreten «: Wie der Großstaat und die Großindustrie sei »die Großwissenschaft, die nicht von Einem geleistet, aber von Einem geleitet wird, ein notwendiges Element« der »Kulturentwicklung, und deren Träger sind die Akademien oder sollten es sein ${ }^{15}$ Damit war der Begriff $»$ Großwissenschaft « geprägt, den Mommsen keineswegs ängstlich und »mournful« verwandte, ${ }^{16}$ sondern vielmehr zukunftsfroh und fortschrittsgläubig.

Halten wir fest: Entscheidenden Anteil an der organisatorischen Modernisierung der Wissenschaften in Deutschland hatten Mommsen und Harnack. Sie sicherten die traditionell führende Rolle der Altertumskunde an der Berliner Akademie bis in die Zeit des Ersten Weltkrieges. Die Altertumswissenschaftler bildeten die weitaus größte Gruppe in der philosophisch-historischen Klasse, stellten die meisten der korrespondierenden und auswärtigen Mitglieder, betreuten die Mehrzahl der Akademieunternehmen und majorisierten mit ihren Beiträgen die Sitzungsberichte. Die altertumswissenschaftlichen Vorhaben dienten anderen Fächern als methodisches und organisatorisches Paradigma; selbst die physikalisch-naturwissenschaftliche Klasse eiferte diesem Vorbild nach. Das deutsche Modell der editorischen Grundlagenforschung wurde zudem im Ausland kopiert, wie ein Blick nach Frankreich, England und Italien zeigt.

\section{Wissenschaftspolitik und die Wissenschaften vom Altertum}

Eine erfolgreiche Universitäts- und Akademiepolitik setzte eine enge Kooperation mit dem vorgeordneten Ministerium der geistlichen, der Unterrichts- und der

15 Mommsen [1890], zitiert nach ders. (1905), 209. Harnacks Antrittsrede und Mommsens Antwort sind abgedruckt in Harnack (1980), Bd. 1, 1-5 (Zitat S. 5) und Adolf von Harnack als Zeitgenosse (1996), Bd. 2, 976-982 (Zitat S. 981 f.)

16 Vgl. Marchand (1996), 75 
Medizinalangelegenheiten voraus. Dabei zählten die Verbindungen zu den einzelnen Ministern, die kamen und gingen, weniger als der Kontakt zu dem Vortragenden Rat und späteren Ministerialdirektor Friedrich Althoff, der seit 1882 der Abteilung IIa der Behörde angehörte, die für Universitäten und wissenschaftliche Anstalten, das höhere Unterrichtswesen, Kunst und Kunstgewerbe zuständig war. Ein Vierteljahrhundert beeinflusste Althoff die staatliche Wissenschafts- und Kulturpolitik nachhaltig und hatte entscheidenden Anteil an der Expansion und Differenzierung des deutschen Hochschul- und Bildungswesens im wilhelminischen Zeitalter. Zentrale Elemente des von ihm geschaffenen >Systems< waren die Professionalisierung und Modernisierung der Hochschulverwaltung, der zunehmende Einfluss der Bürokratie auf die Universitäten, die rigide Kontrolle des Berufungssystems unter bewusster Missachtung der universitären Autonomie, der beschleunigte Ausbau nationaler und internationaler Einrichtungen zur wissenschaftlichen Kooperation, die gezielte Förderung bestimmter Disziplinen an einzelnen Universitäten, die Mobilisierung privater Mittel für die Finanzierung universitärer und außeruniversitärer Forschung und schließlich ein höchst komplexes Netzwerk persönlicher Beziehungen. ${ }^{17}$

Der Althistoriker Mommsen und der Kirchenhistoriker Harnack, die Klassischen Philologen Wilamowitz und Diels, der Klassische Archäologe Ernst Curtius und der Ägyptologe Erman - sie alle wussten um die Bedeutung des Universitätsreferenten Althoff für die Realisierung der großen altertumswissenschaftlichen Unternehmungen. ${ }^{18}$ Auch der Kontakt, den einzelne Vertreter wie Curtius und Harnack zum kaiserlichen Hof unterhielten, konnte ein gutes Verhältnis zu dem Ministerialbeamten nicht ersetzen. Man war sogar bereit, »Willkürregiment « und $\gg$ Favoritenwirtschaft $\ll{ }^{19}$ hinzunehmen, sprich den gouvernementalen Führungsstil Althoffs und die fehlende Transparenz seiner Politik zu tolerieren. Denn nur indem man einen modus agendi mit dem Ministerialbeamten fand, konnten wichtige Forschungsvorhaben realisiert und Einfluss auf die Berufungspolitik ausgeübt werden. Er wolle sich nur ungern mit Althoff überwerfen, sagte Mommsen einem Freund, »denn für viele wissenschaftliche Organisationspläne brauche ich ihn «. ${ }^{20}$ Althoff wiederum brauchte die Professoren der Berliner Universität und die Mitglieder der Preußischen Akademie, um seine wissenschaftspolitischen Ziele durchzusetzen.

17 Da eine neuere Biographie Althoffs fehlt, ist noch immer Sachse (1928) unentbehrlich. Mehrere grundlegende Untersuchungen zur Wissenschaftspolitik und Wissenschaftsorganisation in der Ära Althoff hat in neuerer Zeit Bernhard vom Brocke vorgelegt, vgl. insbesondere vom Brocke (1980) und vom Brocke (1991). Zu Althoffs Bedeutung fur die Altertumswissenschaften vgl. überdies Calder/Košenina (1989).

18 Vgl, zum folgenden Rebenich (1997), 95-116 sowie Rebenich (2002), 145-151.

19 Vgl. Mommsens Brief an Wilamowitz vom 25. Februar 1894, zitiert nach Calder/Kirstein (2003), Nr. 393, 637.

20 Jonas (1917), 36 


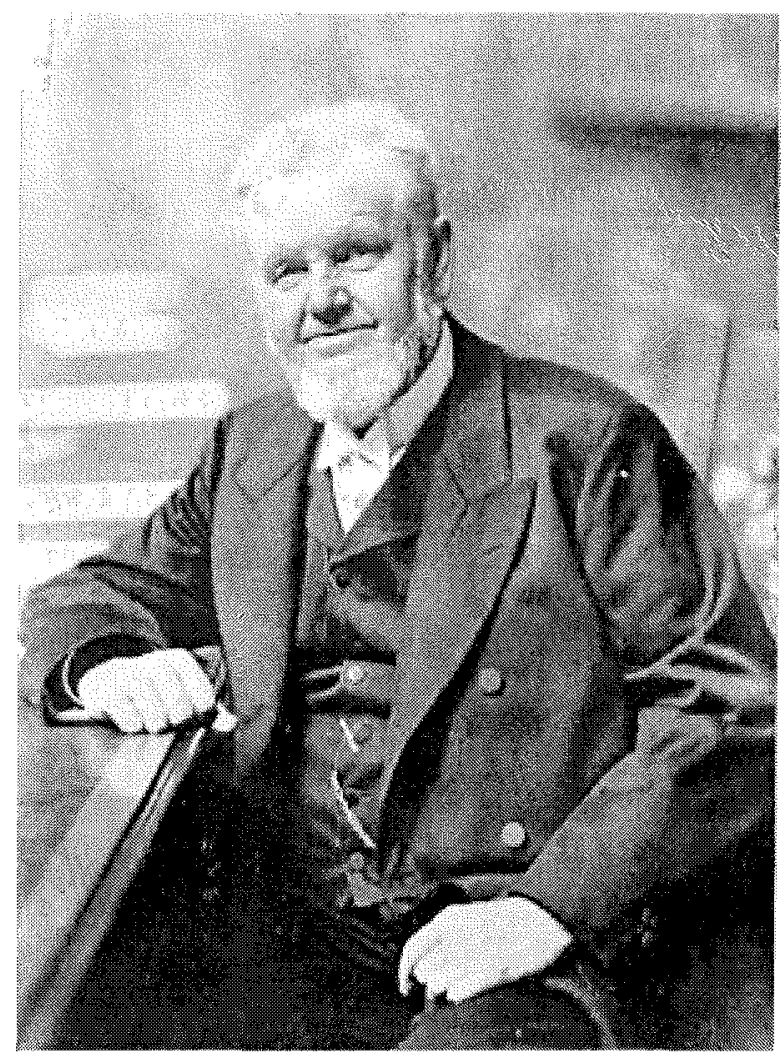

Abb. 2: Friedrich Althoff am Regierungstisch im Preußischen Abgeordnetenhaus, 29. Juli 1907.

Althoff stand in engem Kontakt mit den großen Repräsentanten der einzelnen Disziplinen in Berlin, namentlich mit Gustav Schmoller, Eduard Zeller, Felix Klein, Adolf Harnack, Ulrich von Wilamowitz-Moellendorff und eben Theodor Mommsen. In altertumswissenschaftlichen Berufungsverfahren waren die beiden letztgenannten die einflussreichsten Gutachter. Ihre Bemühungen richteten sich auf das Ziel, die preußische Berufungspolitik in den Altertumswissenschaften zu kontrollieren. Doch erst der maßgeblich von Althoff beeinflusste und gesteuerte personelle Ausbau der preußisch-deutschen Hochschulen und wissenschaftlichen Institutionen ermöglichte zahlreichen jungen Wissenschaftlern eine universitäre Karriere. Pointiert formuliert: Für die Entstehung einer international einflussreichen deutschen Altertumsforschung waren nicht nur die wissenschaftlichen Großprojekte an der Akademie, sondern auch die neuen Rahmenbedingungen einer modernisierten Hochschulverwaltung verantwortlich. 
Wie der Briefwechsel zwischen Mommsen und Althoff zeigt, hatte der Ministerialbeamte immer ein offenes Ohr für altertumskundliche Vorhaben. Man korrespondierte über das Inschriftencorpus und die Münzsammlung, die Reichslimeskommission und die Griechischen Christlichen Schriftsteller. Die Denkschriften, die Mommsen an das Ministerium schickte, um ein Projekt zu begründen oder die Förderung für ein laufendes auszuweiten, gingen grundsätzlich über Althoffs Schreibtisch. Mommsens Plan einer internationalen Assoziation der Akademien stimmte er nachdrücklich zu. Über Leitung, Finanzierung und Aufgaben der Reichsinstitute tauschte man sich ebenso aus wie über drittmittelgeförderte Unternehmungen. Die Akademiestiftungen des Grafen Loubat, von Elisabeth Heckmann-Wentzel und Friedrich Imhoof-Blumer wurden durch Mommsen und Althoff eingerichtet. Doch es ging nicht nur um die Belange der Königlich PreuBischen Akademie der Wissenschaften. Mommsen äußerte sich auch zur Gründung der Biologischen Station Helgoland und wurde bei der Beschaffung eines Refraktors für das Astrophysikalische Institut in Potsdam eingeschaltet. Die Erwerbung der Bibliotheca Meermaniana für die Königliche Bibliothek wurde nur möglich, weil sich unter Mommsens Führung ein Konsortium von Großindustriellen, Bankiers und Gelehrten bereit erklärte, die Sammlung anzukaufen. Mommsens Rat war Althoff in zahllosen Angelegenheiten wichtig: beim Neubau der Königlichen Bibliothek, dem Erwerb von Handschriften, Papyri und Alten Drucken, bei der Reorganisation des Internationalen Leihverkehrs und der Göttinger Gesellschaft der Wissenschaften, bei der Vergabe von Stipendien und der Bewilligung von Förderanträgen, bei der Ehrung von ausländischen Wissenschaftlern und der Schulreform. Auch als der Generaldirektor der türkischen Museen, Hamdi Bey, mehrere Reliefs mit einer Löwenjagd, die deutsche Archäologen auf ihrer Expedition zum Nemrûddagh rechtmäßig erworben hatten, im syrischen Alexandrette entwendete, sollte Althoff helfen.

Welche Schlüsse können wir aus dieser Korrespondenz ziehen? Die sichtbaren Erfolge der preußischen Universitätspolitik, vor allem die institutionelle, personelle und finanzielle Expansion des Wissenschaftssystems waren nicht nur in Mommsens Augen, sondern in der Sicht der meisten Gelehrten Althoff zu verdanken. Man akzeptierte als Voraussetzungen einer erfolgreichen Wissenschaftspolitik die Bürokratisierung, Professionalisierung und Rationalisierung der Verwaltung sowie die hierarchische Struktur des >Systems Althoff<, denn man war überzeugt, dass nur der »Tyrann« Althoff und sein »bürokratischer Caesarismus $\ll^{21}$ den Umbau des deutschen Wissenschaftssystems zu einem modernen, international konkurrenzfähigen Großbetrieb gewährleisten konnten. Die Einsicht, dass man aufeinander angewiesen war, bildete die Grundlage für eine fruchtbare Symbiose in der Wissenschaftspolitik des Deutschen Kaiserreichs und für den Aufstieg der altertumswissenschaftlichen Großwissenschaft.

21 Vgl. Brief Wilamowitz' an Mommsen vom 25. Februar 1894, zitiert nach Calder/Kirstein (2003), Nr. 425, 676. 
Für Mommsen und Curtius, für Harnack und Wilamowitz, für Diels und Erman stand Althoff für eine rational bestimmte Wissenschaftspolitik, die im wissenschaftlichen und nationalen Interesse die Leistungsfähigkeit des preußischen und deutschen Universitätssystems institutionell und personell steigerte und die deutsche Forschung zu internationalem Ansehen führte. Der Gelehrten Ziel war auch Althoffs Ziel: die Weltgeltung der deutschen Wissenschaft im Zeitalter des wilhelminischen Imperialismus.

\section{Wissenschaftstheorie: Die Ordnung der Archive der Vergangenheit}

Die Partikel ớv und die Entelechie des Aristoteles, die heiligen Grotten Apollons und der Götze Besas, das Lied der Sappho und die Predigt der heiligen Thekla, die Metrik Pindars und der Meßtisch von Pompeji, die Fratzen der Dipylonvasen und die Thermen Caracallas, die Amtsbefugnisse der Schultheißen von Abdera und die Taten des göttlichen Augustus, die Kegelschnitte des Apollonios und die Astronomie des Petosiris: alles, alles gehört zur Philologie, denn es gehört zu dem Objekte, das sie verstehen will, auch nicht eines kann sie missen.

In dieser Rede über »Philologie und Schulreform«, die Wilamowitz als Prorektor der Georg-August-Universität zu Göttingen am 1. Juni 1892 hielt, definierte er programmatisch die Aufgabe seiner Disziplin: Die Altertumswissenschaft hatte sich um das griechisch-römische Kulturerbe als Ganzes, um die cognitio totius antiquitatis, der heidnischen wie der christlichen, zu bemïhen. ${ }^{22}$

Mommsen postulierte ebenfalls die Zusammenschau unterschiedlicher Methoden und Quellen, um die traditionelle Zersplitterung der Altertumswissenschaften, wie er sie zu Beginn seiner Laufbahn vorgefunden hatte, zu überwinden. Harnack hat in seiner Trauerrede auf Mommsen als »das Geheimnis seiner wissenschaftlichen Eigenart« bezeichnet, dass dieser »die Aufgaben und Geschäfte der Historie, die sonst verteilt zu sein pflegen, ja die sich auszuschließen scheinen, sämtlich und auf einmal in die Hand nahm und sie nun als der Meister festhielt $\ll$. ${ }^{23}$ Auch Hermann Diels bemühte sich, dem altertumswissenschaftlichen Universalismus gerecht zu werden. Die ganze Kultur des griechisch-römischen Altertums müsse, so schrieb er 1907, »als untrennbare Einheit« umfasst und behandelt werden. ${ }^{24}$

Um zur umfassenden Erkenntnis der Alten Welt vorzudringen, bediente man sich, wie Mommsen im Nachruf auf seinen früh verstorbenen Freund und Lehrer Otto Jahn ausführte, der »streng philologischen Methode «, d. h.

22 Vgl. Wilamowitz-Moellendorff [1901], zitiert nach ders. (1972), 77.

23 Harnack (1903), wiederabgedruckt in Adolf von Harnack als Zeitgenosse (1996), Bd. 2, 1530 1539 (Zitat S. 1533).

24 Diels (1907), 9. 
[...] einfach der rücksichtslos ehrlichen, im großen wie im kleinen vor keiner Mühe scheuenden, keinem Zweifel ausbiegenden, keine Lücke der Überlieferung oder des eigenen Wissens übertünchenden, immer sich selbst und anderen Rechenschaft legenden Wahrheitsforschung. ${ }^{25}$

Also verbrachte man die meiste Zeit seines Forscherlebens mit der Sammlung und der Edition antiker Quellen, auch wenn man diese Arbeit als Qual empfand. Die Selbstverleugnung ging so weit, dass Mommsen spröde chronographische Texte edierte, die er selbst als »chronische Krankheit « bezeichnete. ${ }^{26}$ Doch auf diese Weise wollte man, um Mommsens berühmtes Wort aufzugreifen, »die Archive der Vergangenheit« ordnen, um zur »Grundlegung der historischen Wissenschaft « beizutragen. ${ }^{27}$ Dies war indes kein sonderlich origineller Ansatz. Seit der Renaissance wurden antike Texte ediert, und spätestens seit dem 17. Jahrhundert widmeten sich gelehrte Antiquare den Inschriften und Münzen. Große Sammlungen gab es zuhauf. Mommsens Errungenschaft bestand vielmehr darin, dass er eine neue Methode: die Echtheitskritik, und ein neues Programm: das Totalitätsideal, zusammenführte, um die antike, insbesondere die römische Geschichte zu rekonstruieren. Auch die Altertumsforscher der Generation von Wilamowitz und Diels wollten ihr Scherflein zu ebendieser »Wahrheitsforschung « beitragen.

In der Tradition von Friedrich August Wolf, der die Leistungen der modernen Quellenkritik am Beispiel Homers demonstriert hatte, und von August Boeckh, der gegen die reine »Silben- und Buchstabenkritik« der philologischen Fachidioten polemisiert hatte, verfocht man das Konzept einer umfassenden, verschiedene Einzeldisziplinen integrierenden Altertumswissenschaft. Nicht mehr allein die Textzeugen, sondern die gesamte Hinterlassenschaft der griechischen und römischen Antike wurden von der als historische Wissenschaft verstandenen Philologie in den Blick genommen. Das neue Totalitätsideal erschloss neue Quellen und verlangte nach neuen Methoden.

Boeckh hatte einst als Aufgabe der Altertumswissenschaft »das Erkennen des vom menschlichen Geist Produzierten, d. h. des Erkannten « definiert. ${ }^{28}$ Die Formel von der Erkenntnis des Erkannten machte die Philologie zu einer shistorischen $<$ Wissenschaft. Das »Produzierte« wurde von Boeckh auf alle kulturellen Zeugnisse bezogen, so dass die sprachliche Überlieferung zwar nach wie vor das zentrale, aber nicht mehr das einzige Instrument zum Verständnis der Alten Welt war. Angetrieben wurde Boeckh von der idealistischen Vision, dass die vollständige Erfassung des »Produzierten « - die cognitio totius antiquitatis - die notwen-

25 Vgl. Mommsen (1905), 459

26 Vgl. Mommsens Brief an Wilamowitz vom 16. Juli 1893, zitiert nach Calder/Kirstein (2003), Nr. 379/380, 617 .

27 Vgl. Mommsen (1858), zitiert nach ders. (1905), 37.

28 Boeckh (1886/1966), 10. 
dige Grundlage der wahrheitsgetreuen Rekonstruktion der historischen Wirklichkeit sei.

Mommsen teilte zwar diese Auffassung, veränderte aber die Boeckhsche Altertumswissenschaft in inhaltlicher und organisatorischer Hinsicht grundlegend. Nach industriellem Vorbild schuf er einen Großbetrieb der Forschung, in der der Mensch der Wissenschaft, nicht die Wissenschaft dem Menschen diente. Erst durch die von ihm geleiteten >Langzeitunternehmen< der Akademie erhielt Boeckhs Totalitätsgedanke ubiquitäre Bedeutung. Jetzt wurde das gesamte erhaltene Quellenmaterial aus der Antike mit beispiellosem Aufwand gesammelt, geordnet und ediert. Literarische Texte, Inschriften, Papyri, Münzen und archäologische Überreste wurden erfasst. Damit hoffte man, wie Wilamowitz einmal schrieb, die griechisch-römische Kultur »in ihrem Wesen und allen Äußerungen ihres Lebens « erfassen zu können. ${ }^{29}$

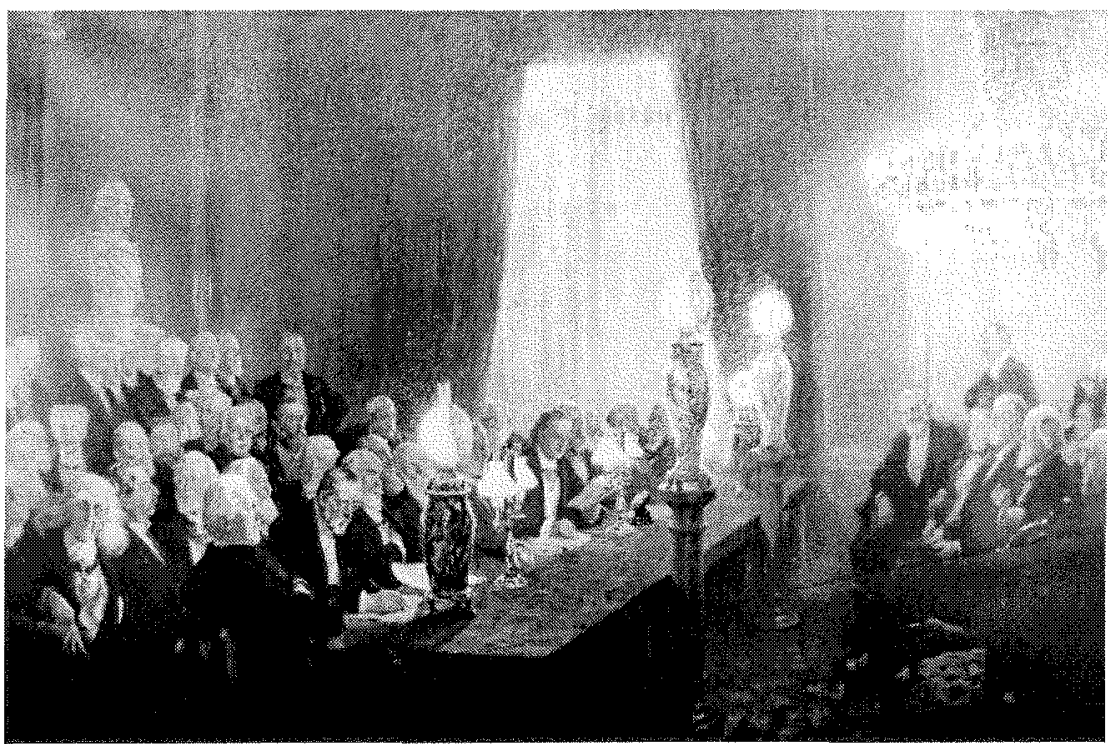

Abb. 3: Die letzte Sitzung der Akademie der Wissenschaften in Berlin im alten Gebäude 1903. Links vorne (dem Betrachter zugewandt) sitzt Theodor Mommsen, schräg hinter ihm Adolf Harnack, unmittelbar vor ihm Ulrich von Wilamowitz-Moellendorff.

Mommsen, von der Rechtswissenschaft kommend, wandte sich der gesamten römischen Geschichte zu, Wilamowitz der griechischen Literatur in ihrer ganzen Breite von den Anfängen bis in die christliche Spätantike. Hermann Diels machte

29 Wilamowitz-Moellendorff (1927), 1 
sich um die griechischen Philosophen und die griechischen Ärzte verdient, Ernst Curtius edierte griechische Inschriften und führte in Olympia die erste systematische Großgrabung europäischer Staaten im Mittelmeerraum durch, ${ }^{30}$ Harnack wollte durch die Herausgabe der Kirchenväter zum Proprium der christlichen Religion, dem vom Ballast der antiken Überlieferung befreiten Evangelium Jesu Christi vordringen, und Adolf Erman schuf eine "ägyptische Altertumswissenschaft von der Sprache und den Sachen « nach dem Vorbild der griechischrömischen Altertumskunde. ${ }^{31}$ Selbst die Verzettelung des Materials wurde beim Wörterbuch der altägyptischen Sprache nach den Vorgaben des 1893 begonnenen Thesaurus linguae Latinae durchgeführt. ${ }^{32}$

Über die theoretischen Grundannahmen ihres Wissenschaftsverständnisses reflektierten Mommsen und seine Zeitgenossen nicht. Die Bemühungen eines Friedrich August Wolf oder August Boeckh um eine wissenschaftliche Theorie und universale Methodologie wurden nicht fortgesetzt. Man beschränkte sich auf die hochspezialisierten Operationen der Quellenkritik und der Hermeneutik. An die Stelle einer philosophisch begründeten Wissenschaftstheorie trat die Reflexion über die Organisation einer in Universitäten und Akademien institutionalisierten Altertumswissenschaft. Hermann Diels verfasste 1906 im ersten Band der von Paul Hinneberg herausgegebenen Reihe »Kultur der Gegenwart« einen Beitrag über $\gg$ Die Organisation der Wissenschaft $\ll ; 3$ ein Jahr zuvor hatte bereits Adolf Harnack in den Preußischen Jahrbüchern über den »Großbetrieb der Wissenschaft « nachgedacht. ${ }^{34}$ Hier wurden in der Tat großartige Erfolge erzielt. Die Gemeinschaftsunternehmen erschlossen systematisch das Erbe der Alten Welt. Die Leistungsfähigkeit der historisch-kritischen Methode war eindrucksvoll, auch wenn Heuristik und Interpretation immer öfter auseinander fielen. Ein analytischhistorischer Empirismus erhob selbstbewusst sein Haupt. Fortschrittsgläubigkeit und Wissenschaftsoptimismus kennzeichneten eine professionalisierte Altertumskunde, deren bedeutendste Vertreter bis an ihr Lebensende auf die Zielgerichtetheit und Vernünftigkeit der Weltgeschichte vertrauten - und von der Überlegenheit ihrer Wissenschaft überzeugt waren. So wunderte sich Mommsen, dass ein so gescheiter Kopf wie Hermann von Helmholtz an naturwissenschaftlichen Fragen Gefallen finden könne. ${ }^{35}$ Wilamowitz wollte gar das Helmholtz-Denkmal vor der Friedrich-Wilhelms-Universität verschwinden lassen, da es sich nicht schicke,

30 Vgl. z. B. Marchand (1996), 75 ff. sowie den Beitrag von Henning Wrede in diesem Band.

31 Grapow (1955), $10(=[1986], 12)$.

32 Rebenich (2006).

33 Diels (1906).

34 Harnack (1905), wiederabgedruckt in Adolf von Harnack als Zeitgenosse (1996), Bd. 2, 10091019 (Zitat S. 1014).

35 Vgl. Diels (1907), 5 
dass sich die Naturwissenschaft einen Herrschaftsplatz anmaße. ${ }^{36}$ Hermann Diels polemisierte gegen eine in den Sitzungsberichten der Akademie erschienene Publikation des Mineralogen Karl Klein »Über Buntkupfererz aus Tyrol : »Nomen et omen habet! Und mit solcher Sorte soll man zusammen arbeiten! «"

\section{Die Antike als Einheit: Das verlorene Ideal}

Die Historisierung des Altertums im Großbetrieb der Wissenschaften hatte mit der klassizistischen Entrückung und neuhumanistischen Idealisierung der Antike nichts mehr gemein. Boeckh hatte nie einen Zweifel daran gelassen, dass die Kultur der Griechen und Römer die Grundlage der gesamten Bildung sei. Eine solche normative Betrachtung der Antike war seinen Nachfolgern im Kaiserreich fremd. Ihr moderner Realismus zerstörte die Sonderstellung der Griechen, die dem deutschen Bildungsbürger zur lieben Gewissheit geworden war. ${ }^{38}$

Die enorme Verbreiterung der Quellenbasis hatte zugleich weitreichende Folgen für die Altertumswissenschaften. Zum einen hatte die divinatorische Kraft des Geistes, die Boeckh noch beschworen hatte, ausgedient. Strenge Urkundlichkeit wurde gefordert, jede These musste an den Quellen überprüft werden. Zum anderen rückte eine Vielzahl von Einzelproblemen in den Vordergrund. Jeder Erkenntniszuwachs, war er auch noch so klein, diente der wissenschaftlichen Selbstbestätigung. Platons Ideenlehre fand ebensolche Aufmerksamkeit wie seine Nachtuhr. In solchen Untersuchungen manifestierte sich, um eine Formulierung Walter Burkerts aufzugreifen, »die Selbstversponnenheit einer Wissenschaft, die sich selbst unanfechtbar als Höchstzweck empfand $\ll .{ }^{39}$ Während die >Heroen noch in der Lage waren, die Ergebnisse ihrer weitverzweigten und komplexen Detailstudien zu überblicken und in großen Synthesen zu bündeln, vermochten sich ihre >Epigonen $<$ immer weniger aus der Isolation einer hochspezialisierten Realienforschung zu befreien. ${ }^{40}$ Schließlich beschleunigte sich die organisatorische und institutionelle Differenzierung der Altertumsforschung analog zu anderen Wissenschaften. Die enorme Vergrößerung der Aufgabengebiete hatte die disziplinäre Verselbständigung der Lateinischen und Griechischen Philologie, der Klassischen Archäologie, der Alten Geschichte und der Hilfswissenschaften zur Folge. Diese Entwicklung konnte auch die Konzeption einer alle Einzeldisziplinen umfassenden klassischen Altertumswissenschaft, die Wilamowitz entwickel-

36 Wilamowitz-Moellendorff (1929), 293. Man beachte, dass Wilamowitz nicht einmal den Namen des weltberühmten Naturwissenschaftlers richtig zu schreiben vermochte: statt Helmholtz ist Helmholz zu lesen.

37 Vgl. Rebenich (1999b), 91.

38 Vgl. Stähli (2001).

39 Burkert (1969), XIII.

40 Zur Differenzierung zwischen Heroen und Epigonen vgl. Unte (2003), IV. 
te, ebenso wenig aufhalten wie der von Eduard Meyer unternommene Versuch, Alte Geschichte als Teil der Universalgeschichte in Forschung und Lehre darzustellen. Damit wurde die Antike als fächerübergreifendes Ideal zerstört und die Desintegration der einzelnen Fachbereiche eingeleitet. Die Modernisierung der altertumskundlichen Fächer stand im offenen Widerspruch zu der immer wieder beschworenen Einheit der Altertumswissenschaften.

Die explosionsartige Mehrung des Wissens und die Pluralisierung der Wertvorstellungen führten in der zweiten Hälfte des 19. Jahrhunderts zu einer tiefgreifenden Verunsicherung. Zunehmend wurde Kritik an dem >Positivismus< einer in sich selbst versponnenen Tatsachenforschung und dem Relativismus einer Wissenschaft geäußert, die alle Werte unterschiedslos historisiere und komplexe gesellschaftliche Strukturen nur ungenügend $\mathrm{zu}$ beschreiben vermöge. Das böse Wort vom >Historismus sollte schließlich die Runde machen. ${ }^{41}$ Der vermeintliche Objektivismus der Altertumswissenschaften wurde als steril und lebensfeindlich empfunden. Eine Überfülle von Material, so lautet ein häufig zu vernehmender Vorwurf, werde angehäuft, ohne dass man über die Notwendigkeit und Funktion solcher Sammlungen Rechenschaft gebe. Jacob Burckhardt kritisierte in seinen Vorlesungen »Über das Studium der Geschichte« die werterelativierende Wirkung einer auf individualisierendem Verstehen gegründeten Geschichtswissenschaft, die die Vergangenheit um ihrer selbst willen erforsche, ohne dass nach der lebenspraktischen Relevanz historischer Erkenntnisse gefragt werde. Friedrich Nietzsche attackierte 1874 in seiner zweiten Unzeitgemäßen Betrachtung über den Nutzen und Nachteil der Historie für das Leben den Fortschrittsoptimismus seiner Kollegen, die aus der Vergangenheit die Gegenwart verstehen wollten. Tatsächlich jedoch könne die historische Wissenschaft durch die Zerstörung aller geschichtlichen Normen keine konkrete Hilfe für die Lebensgestaltung geben. Eben deshalb entwarf Nietzsche gegen die theoretischen und methodischen Standards der zeitgenössischen Altertums- und Geschichtswissenschaft das Konzept einer dem Leben dienenden Historie.

Doch auch von anderer Seite regte sich Kritik. Johann Gustav Droysen, der sich intensiv um eine Theorie der Geschichte bemühte, warnte davor, dass die Studenten nur noch »zum Spezialisten« reiften, weil sie sich in der »Fabrikarbeit für die Monumenta oder Urkundenbücher « verschlissen. "Innere Spannkraft, geistige Erhebung, schöpferisches Denken « würden in den großen wissenschaftlichen Unternehmungen nicht geschult. ${ }^{42}$ Und Heinrich von Treitschke schrieb 1885 an seine Frau: $\gg$ Unter den jungen Historikern geht die Erkenntnis, dass die Geschichte Darstellung des Lebens ist, schon fast verloren über der Tüftelei der Quellenforschung. ${ }^{43}$ Selbst Mommsen glaubte, ein »zugleich geniales und methodisches Werk« werde »tausend Male mehr nützen als alles Erbsenwerfen

41 Vgl, Rebenich (2000) mit weiterer Literatur.

42 Zitiert nach Hübner (1929), 941 f.

43 Cornicelius (1920), $585 \mathrm{f}$. 
und Schwärmerabbrennen«. Doch auch er tat nichts gegen »jene Urkundskompilatoren und Spezialforscher, die auf zusehends enger gezogenen Parzellen ständig tiefer gruben $\ll$. ${ }^{44}$ Und Wilamowitz beschwor unablässig die Idealität der klassischen Antike und das küntlerisch produktive Individuum, blieb aber in seinen Forschungen dem Historismus verhaftet.

Die Großforschung der Akademie repräsentierte das Selbstverständnis der altertumswissenschaftlichen Disziplinen in Deutschland: Das kleinste Fragment war des Sammelns wert, da es ein potentielles Objekt künftiger Erkenntnis sein konnte. Die Erforschung und Systematisierung der Überlieferung blieb die zentrale Aufgabe der historischen Fächer. Dafür nahm man die radikale Relativierung der individuellen Forschungsleistung in Kauf. Aus dem Gelehrten wurde der fleiBige Diener der Wissenschaft, ${ }^{45}$ der Arbeiter und Kärrner, der sich nun in einer säkularisierten Form der Askese zu bewähren hatte - in Mommsens Worten, gesprochen am Gedächtnistag des großen Universalisten Leibniz am 4. Juli 1895:

Die Wissenschaft allerdings schreitet unaufhaltsam und gewaltig vorwärts; aber dem emporsteigenden Riesenbau gegenüber erscheint der einzelne Arbeiter immer kleiner und geringer. [...] Unser Werk lobt keinen Meister und keines Meisters Auge erfreut sich an ihm; denn es hat keinen Meister und wir sind alle nur Gesellen. [...] Wir klagen nicht und beklagen uns nicht: die Blume verblüht, die Frucht muß treiben. Aber die Besten von uns empfinden, daß wir Fachmänner geworden sind. ${ }^{46}$

\section{Versuch einer Bilanz}

Konsequent stellten die Altertumswissenschaftler der Akademie ihre Schaffenskraft in den Dienst eines Wissenschaftsverständnisses, das den antiquarischen Vollständigkeitsanspruch absolut setzte. Ihre Arbeit trug reiche Frucht. Die von ihnen initiierten und geleiteten Großprojekte der Berliner Akademie bildeten und bilden noch immer die unverzichtbare Quellengrundlage für eine Vielzahl altertumswissenschaftlicher Studien. Hier wurden methodische und organisatorische Standards der Fächer gesetzt, die nach wie vor unverzichtbar sind. Ein Beispiel soll genügen: Das lateinische Inschriftencorpus verschaffte der Forderung nach Autopsie der epigraphischen Zeugnisse universelle Gültigkeit und begründete die internationale Kooperation von Epigraphikern und Altertumswissenschaftlern. Gewiss, manche Projekte gingen nur schleppend voran, und andere mussten ganz fallengelassen werden; und es ist kein Privileg unserer Zeit, dass Forschungsgelder für letztlich undurchführbare Unternehmen verschwendet werden. Erinnert sei nur an Mommsens letztes Großprojekt - die Prosopographia Imperii Romani

44 Zitiert nach Brandt (2004), 149.

$45 \mathrm{Zu}$ diesem Begriff vgl. Rebenich (1997), 653.

46 Mommsen (1895), zitiert nach ders. (1905), 196-198. 
saec. IV.V.VI. ${ }^{47}$-, das ein personenkundliches Arbeitsinstrument für Profan- und Kirchenhistoriker sowie Theologen und Philologen zuwege bringen wollte. Das Vorhaben scheiterte letztlich an dem zu weit gesteckten Ziel, das Mommsen zu verantworten hatte. Es wurde 1933 eingestellt, nachdem 75000 Zettel verfasst, ein Projektleiter verschlissen und fast 100000 Mark verbraucht worden waren. Dennoch bleiben die altertumswissenschaftlichen Akademieunternehmen eine Erfolgsgeschichte - auch über das 19. Jahrhundert hinaus. Hier wurden neue Quellengattungen erschlossen, Hunderte von Texten und Abertausende von Inschriften ediert und unverzichtbare Nachschlagewerke in Angriff genommen. Es wurden mithin Grundlagen gelegt, auf denen unsere heutige Forschung in vielerlei Hinsicht aufbaut.

Ein Weiteres kommt hinzu: In den Unternehmen wurde ein großer Teil des wissenschaftlichen Nachwuchses geschult. Auch wenn einzelne Projektleiter, allen voran Mommsen, Mitarbeiter für eigene Forschungen ausnutzten und dann »schroff bei Seite warfen ${ }^{48}$ diente die Akademie schon vor der Einrichtung wissenschaftlicher Beamtenstellen im Jahr 1900 (im Zusammenhang mit der Zweihundertjahrfeier) der wissenschaftlichen Aus- oder Weiterbildung vielversprechender junger Forscher, denen konkrete Arbeitsaufträge - von der Kollation bis zur Edition - anvertraut wurden. ${ }^{49}$

Doch der Großbetrieb der Altertumswissenschaft zerstörte zugleich den neuhumanistischen Glauben an die Normativität der Alten, und er gab keine Antwort auf die immer drängendere Frage, wie Wissenschaft und Leben zu verbinden seien. So schwand seit dem Ende des 19. Jahrhunderts die Zuversicht, dass sich wissenschaftlicher Erkenntniszuwachs in immer größeren und detaillierteren Quelleneditionen spiegele. Selbst Wilamowitz sprach verächtlich von Mommsens lateinischem Inschriftenwerk als dis manibus-Wissenschaft. ${ }^{50}$ Doch was hatte er anzubieten? Die »ethische Verhaltenheit« der protestantischen Askese und die Selbstinszenierung in der Goethemaske, wie Carl Schmitt bemerkte. ${ }^{51}$ Sein ungarischer Kommilitone polemisierte gegen »die Mittelschullehrer- und Philologenantike« mit ihren »gesetzten leblosen Forschungen « und wollte zu einer humanistischen Wissenschaft zurückfinden, die es den Menschen ermögliche, »ein Verhältnis zu allem zu finden, was hier auf Erden Großes und Schönes geschaffen worden ist ${ }^{52}$ Diese Generation wandte sich daher wieder Herder, Humboldt und Hölderlin zu und las statt Wilamowitz lieber Burckhardt, Nietzsche und George.

Die altertumskundlichen Unternehmungen verschanzten sich hingegen hinter den hohen Mauern der Akademie. Man vertraute darauf, dass alles beim Alten

47 Vgl. Rebenich (1997), 247-326.

48 So Wilamowitz in einem Brief an Edward Fitch vom 12. Juni 1907, vgl. Calder (1983), 76.

49 Vgl. Rebenich (1997), 210-223 und Rebenich (2002), 158-164.

50 Braun/Calder/Ehlers (1995), 232 mit Anm. 831.

51 Vgl. Tommissen (1990), $18 \mathrm{f}$.

52 Vgl Hatvany (1908/1986), 24 f. und 113. 
bleiben würde. Die dominierende Stellung der Altertumswissenschaftler in der Akademie und die kontinuierliche Arbeit in den Projekten stärkten die Kräfte der Beharrung. Neue Fragestellungen wurden ebenso wenig aufgegriffen wie innovative Formen der Forschungsorganisation. Man hatte sich in einem Wissenschaftsbetrieb behaglich eingerichtet, den Ernst Troeltsch treffend charakterisierte: »Völlig relativistische Wiedererweckung beliebiger vergangener Bildungen mit dem lastenden und ermüdenden Eindruck historischer Allerweltskenntnis und skeptischer Unproduktivität für die Gegenwart. ${ }^{53}$ An dem Großbetrieb der Altertumswissenschaften lässt sich exemplarisch das Dilemma der historistischen Altertumskunde darstellen: Die ungeheure Ausweitung des historischen Wissens in Breite und Tiefe hatte die Relativierung ebendieses Wissens zur Folge.

Seit dem Ersten Weltkrieg wurde die Kritik an einem vermeintlich degenerierten Historismus, an dem epigonalen Charakter eines reinen Forschungspositivismus und der Verabsolutierung individualistischer Subjektivität lauter. ${ }^{54}$ Zahlreiche neue Ansätze versuchten, den >historischen Positivismus^ zu überwinden und die Kluft zwischen Wissenschaft und Leben zu überbrücken. Der Mehrzahl dieser Konzepte ist gemeinsam, dass sie die Antike als sinnstiftende historische Größe rehabilitieren wollten. Werner Jaeger etwa, Wilamowitz' Nachfolger auf dem Lehrstuhl für Klassische Philologie und seit 1924 ordentliches Mitglied der Berliner Akademie, begründete mit seinem »Dritten Humanismus « ein KlassikKonzept >jenseits des Historismus<, das die griechische Antike fokussierte, sich inhaltlich durch den paideia-Begriff bestimmte und Geschichte als teleologischen Prozess definierte. Ein tief sitzendes Krisenbewusstsein, die Konkurrenz wissenschaftlicher und politischer Leitsysteme, antidemokratische und antiparlamentarische Überzeugungen, die schwindende Bedeutung der Antike und - last, but not least - ein antihistoristischer Reflex ließen allerdings einzelne Gelehrte auf ihrer Suche nach einem neuen Bild der Antike faschistische und nationalsozialistische Ideologeme rezipieren.

Die Altertumskunde der Berliner Akademie, die strenge Objektivität und Rationalität einforderte, ging indes mit den irrationalen und kulturkritischen Ideologemen des Nationalsozialismus nicht konform. ${ }^{55}$ Man war nicht willens, den Erwartungen der neuen Zeit zu entsprechen, die der bayerische Kultusminister Hans Schremm prägnant formuliert hatte: Es komme nicht mehr darauf an festzustellen, »ob etwas wahr ist, sondern ob es im Sinne der nationalsozialistischen Revolution ist ${ }^{56}{ }^{56}$ Die auf der strengen philologischen Methode beruhende »rücksichtslos ehrliche, [...] immer sich selbst und anderen Rechenschaft legende Wahrheitsforschung ${ }^{57}$ konnte hier nicht willfahren. Wie bereits der in den zwan-

53 Zitiert nach Timpe (1973), wiederabgedruckt in Nippel (1993), 361.

54 Zum folgenden vgl. Rebenich (2001c) und Rebenich (2008/2009).

55 Vgl. Rebenich (2001a).

56 Zitiert nach Erdmann (1980), 171.

57 Mommsen (1905), 459. 
ziger Jahren propagierte Paradigmenwechsel in den Altertumswissenschaften der Akademie kaum Anklang gefunden hatte, so stießen die in den dreißiger Jahren unternommenen Versuche, die Altertumskunde zu nazifieren, bei den Mitgliedern und Mitarbeitern in der Akademie mehrheitlich auf Ablehnung. Doch das methodisch innovative Potential war aufgebraucht. Man begnïgte sich allenthalben mit gediegener und zuverlässiger Grundlagenarbeit, feierte das humanistische Erbe Europas und beschwor die Fiktion der apolitischen res publica litterarum auch dann noch, als die Nazis schon längst jüdische Mitglieder und Mitarbeiter aus der Akademie vertrieben hatten. Der Verlust an hochqualifizierten Wissenschaftlern durch die Nazi-Barbarei und durch den Zweiten Weltkrieg ist ein entscheidender Grund für den Niedergang der Altertumswissenschaften, nicht nur an der Berliner Akademie.

Die Mehrzahl der altertumswissenschaftlichen Großunternehmungen überstand die politischen Wirren der Nachkriegszeit, die Reorganisation der Akademie in der Deutschen Demokratischen Republik und die Umbrüche der Wendeund Nachwendezeit. Erst eine neue Akademiepolitik, die durch Reformrhetorik und Evaluationseuphorie gekennzeichnet ist, hat am Ausgang des 20. und zu Beginn des 21. Jahrhunderts die Langzeitforschung als fragwürdigen Teil des historistischen Erbes eines vergangenen Zeitalters relativiert. Heftig wurde gegen die »Riesenschildkröten« polemisiert, und die geisteswissenschaftlichen Vorhaben zur Materialerschließung haben in der heutigen Wissenschaftspolitik keine Konjunktur. ${ }^{58}$ Die apotropäische Beschwörung der Archegeten der modernen Altertumskunde hilft in dieser Situation nicht, will man das Überleben der Projekte sichern. Es sind vor allem drei Gründe zu nennen, die für den Fortbestand der altertumswissenschaftlichen Langzeitunternehmen sprechen.

Zum einen sind sie wie bereits im 19. Jahrhundert maßgebliche Instrumente altertumswissenschaftlicher Forschung. Günther Klaffenbachs Wort von 1957 gilt auch heute noch:

Aber man darf gewiß sein, daß sich gegenüber der wachsenden Vielzahl von Sonderpublikationen das Bedürfnis nach wenigen großen, zusammenfassenden Editionen, an die sich jeder Vertreter der Altertumswissenschaft ohne langes Suchen und Fragen wenden kann, wieder durchsetzen wird. ${ }^{59}$

Die Akademievorhaben garantieren mithin den Zuwachs an Erkenntnischancen in den Altertumswissenschaften.

Zum anderen finden sich nur an den Akademien die organisatorischen und personellen Voraussetzungen für langfristig angelegte Unternehmen zur Quellenerschließung. Universitäre Institute und »Exzellenzzentren« können diese Aufgabe nur unzureichend übernehmen; ihr Augenmerk gilt nicht der vermeintlich wenig innovativen editorischen Grundlagenforschung, sondern anderen finanzintensiven Förderinstrumenten wie Sonderforschungsbereichen, Graduiertenkol-

58 Vgl. Jungen (2006).

59 Klaffenbach (1966), 20. 
legs und Exzellenzclustern, die zeitlich befristet sind, Interdisziplinarität einfordern und auf rasche Ergebnisse setzen.

Schließlich ist die Bedeutung der Langzeitunternehmen für die Nachwuchsrekrutierung hervorzuheben. Allerdings kann es nicht mehr deren Ziel sein, künftige Hochschullehrer in den altertumskundlichen Hilfswissenschaften zu unterrichten, sondern vielmehr den eigenen Nachwuchs auszubilden; denn welcher Graduierte der Alten Geschichte kann heute noch griechische oder lateinische Inschriften edieren, welcher Absolvent der Klassischen Philologe griechische medizinische Texte oder welcher frisch examinierte Theologe griechische christliche Schriftsteller? Der Rückgang altsprachlicher Kenntnisse und die Marginalisierung der Sprachenausbildung in zahlreichen modularisierten Studiengängen wird die Bedeutung der Unternehmen für die Schulung von altertumswissenschaftlichen Spezialisten noch stärker hervortreten lassen, zumal die Universitäten, die immer kurzfristiger planen müssen, diese Aufgabe nicht mehr übernehmen können.

Darïber hinaus lehrt der Rekurs auf die Geschichte der altertumswissenschaftlichen Großwissenschaft, dass man sich vor modischen Aktualisierungen und politischen Instrumentalisierungen hüten sollte. $\gg$ Die Antike als Einheit und als Ideal ist dahin; die Wissenschaft selbst hat diesen Glauben zerstört. $\ll^{60}$ Zumindest darin ist Wilamowitz zuzustimmen. Wir haben uns unwiderruflich von einem humanistischen Geschichtsbewusstsein emanzipiert, das sich mit der Überlieferung identifizierte. ${ }^{61}$ Die antike Tradition kann nur geschichtlich verstanden werden. In dieser Situation griffe es zu kurz, über eine Politik zu klagen, die die einzelnen Wissenschaften nur noch nach sozialen und ökonomischen Parametern bewertet, und eine Öffentlichkeit zu verurteilen, die das Erbe der Alten Welt nicht mehr als selbstverständlichen Bestandteil ihrer Kultur versteht. Die altertumswissenschaftlichen Unternehmungen an den Akademien haben dem stetig wachsenden Legitimationsdruck standzuhalten und müssen ihre Bedeutung für die geisteswissenschaftliche Grundlagenforschung selbstbewusst artikulieren.

\section{Literatur}

Adolf von Harnack als Zeitgenosse. Reden und Schriften aus den Jahren des Kaiserreichs und der Weimarer Republik, hg. v. Kurt Nowak, 2 Bde., Berlin/New York 1996.

Boeckh, August, Encyklopädie und Methodologie der philologischen Wissenschaften, hg. v. Ernst Bratuscheck, 2. Aufl. bes. v. Rudolf Klußmann, Leipzig 1886 [Nachdruck. Darmstadt 1966].

60 Wilamowitz-Moellendorff [1901], zitiert nach ders. (1972), 79.

61 Vgl. Timpe (1973), wiederabgedruckt in Nippel (1993), 368. 
Brandt, Hartwin, »Mommsens Sicht von Münze und Geld in der römischen Kaiserzeit und Spätantike«, in: Geldgeschichte versus Numismatik. Theodor Mommsen und die antike Münze. Kolloquium aus Anlaß des 100. Todesjahres von Theodor Mommsen (1817-1903) an der Johann Wolfgang Goethe-Universität Frankfurt am Main (1.4. Mai 2003) veranstaltet durch Fundmünzen der Antike, Akademie der Wissenschaften und der Literatur Mainz, und Griechisches Münzwerk, Berlin-Brandenburgische Akademie der Wissenschaften, hg. v. Hans-Markus von Kaenel/Maria R. Alföldi/ Ulrike Peter/Holger Komnick, Berlin 2004, 149-155.

Braun, Maximilian/Calder, William M. III/Ehlers, Dietrich (Hg.), »Lieber Prinz». Der Briefwechsel zwischen Hermann Diels und Ulrich von Wilamowitz-Moellendorff (1869-1921), Hildesheim 1995.

Burkert, Walter (Hg.), Hermann Diels. Kleine Schriften zur Geschichte der antiken Philosophie, Darmstadt 1969.

Calder, William M. III (Hg.), Ulrich von Wilamowitz-Moellendorff. Selected Correspondence 1869-1931, Neapel 1983 (Antiqua, 23).

Calder, William M. III/Fowler, Robert L. (Hg.), The Preserved Letters of Ulrich von Wilamowitz-Moellendorff to Eduard Schwartz, München 1986 (Sitzungsberichte der Bayerischen Akademie der Wissenschaften, Philosophisch-historische Klasse, Jg. 1986, Nr. 1).

Calder, William M. III/Kirstein, Robert (Hg.), »Aus dem Freund ein Sohn". Theodor Mommsen und Ulrich von Wilamowitz-Moellendorff. Briefwechsel 1872-1903, 2 Bde., Hildesheim 2003.

Calder, William M. III/Košenina, Alexander (Hg.), Berufungspolitik innerhalb der Altertumswissenschaft im wilhelminischen Preußen. Die Briefe Ulrich von WilamowitzMoellendorfs an Friedrich Althoff (1883-1908), Frankfurt am Main 1989.

Cornicelius, Max (Hg.), Heinrich von Treitschke. Briefe, Bd. 3.2, Leipzig 1920.

Diels, Hermann, »Die Organisation der Wissenschaft«, in: Die Kultur der Gegenwart, hg. v. Paul Hinneberg, Bd. 1.1, Berlin/Leipzig 1906, 591-650.

Diels, Hermann, »Die Einheitsbestrebungen der Wissenschaft «, in: Internationale Monatsschrift für Wissenschaft, Kunst und Technik 1 (1907), 3-10.

Erdmann, Karl Dietrich, Deutschland unter der Herrschaft des Nationalsozialismus 19331939, 9. Aufl. München 1980 (Gebhardt, Handbuch der deutschen Geschichte, 20).

Grapow, Hermann, Worte des Gedenkens an Adolf Erman anläßlich seines hundertsten Geburtstages am 31. Oktober 1954, Berlin 1955 (Sitzungsberichte der Deutschen Akademie der Wissenschaften zu Berlin, Klasse für Sprachen, Literatur und Kunst, Jg. 1954, Nr. 3) [Nachdruck in: Adolf Erman. Akademieschriften (1880-1928), Bd. 1: 1880-1910, Leipzig 1986, 5-20].

Harnack, Adolf, Geschichte der Königlich Preußischen Akademie der Wissenschaften zu Berlin, 3 Bde. in 4, Berlin 1900.

Harnack, Adolf, Rede bei der Begräbnisfeier Theodor Mommsens am 5. November 1903, Leipzig 1903 [Nachdruck in: Adolf von Harnack als Zeitgenosse. Reden und Schriften aus den Jahren des Kaiserreichs und der Weimarer Republik, hg. v. Kurt Nowak, Bd. 2, Berlin/New York 1996, 1530-1539].

Harnack, Adolf, »Vom Großbetrieb der Wissenschaft«, in; Preußische Jahrbücher 119 (1905), 193-201 [Nachdruck in: ders., Aus Wissenschaft und Leben. Reden und Aufsätze, N. F., Bd. 1, Gießen 1911, 10-20]. 
Harnack, Adolf von, Kleine Schriften zur Alten Kirche, Bd. 1: Berliner Akademieschriften 1890-1907, Bd. 2: Berliner Akademieschriften 1908-1930, hg. v. Jürgen Dummer, Leipzig 1980.

Hatvany, Ludwig, Die Wissenschaft des Nicht Wissenswerten, Leipzig 1908 [2. Aufl. Berlin 1911; Nachdruck mit einem Vorwort von Hugh Lloyd-Jones, Oxford 1986].

Hirschfeld, Otto, »Gedächtnisrede auf Theodor Mommsen«, in: Abhandlungen der Königlich Preußischen Akademie der Wissenschaften, Philosophisch-historische Klasse, Jg. 1904, 1025-1060 [Nachdruck in: ders., Kleine Schriften, Berlin 1913, 931-965].

Hübner, Rudolf (Hg.), Johann Gustav Droysen. Briefwechsel, Bd. 2: 1851-1884, Stuttgart 1929 (Deutsche Geschichtsquellen des 19. Jahrhunderts, 26).

Jonas, Fritz, Erinnerungen an Theodor Mommsen zu seinem hundertjährigen Geburtstage, Berlin o. J. [1917].

Jungen, Oliver, »Was lange währt. Verzettelung: Die Langfristforschung deutscher Akademien «, in: Frankfurter Allgemeine Zeitung, 12. August 2006.

Kaenel, Hans-Markus von/Alföldi, Maria R./Peter, Ulrike/Komnick, Holger (Hg.), Geldgeschichte versus Numismatik. Theodor Mommsen und die antike Münze. Kolloquium aus Anlaß des 100. Todesjahres von Theodor Mommsen (1817-1903) an der Johann Wolfgang Goethe-Universität Frankfurt am Main (1.-4. Mai 2003) veranstaltet durch Fundmünzen der Antike, Akademie der Wissenschaften und der Literatur Mainz, und Griechisches Münzwerk, Berlin-Brandenburgische Akademie der Wissenschaften, Berlin 2004.

Kirsten, Christa, Die Altertumswissenschaften an der Berliner Akademie. Wahlvorschläge zur Aufnahme von Mitgliedern von F. A. Wolf bis zu G. Rodenwaldt 1799-1932, Berlin 1985 (Studien zur Geschichte der Akademie der Wissenschaften der DDR, 5).

Klaffenbach, Günther, Griechische Epigraphik, 2. Aufl. Göttingen 1966 (Studienhefte zur Altertumswissenschaft, 6).

Kocka, Jürgen (Hg.), Die Königlich Preußische Akademie der Wissenschaften zu Berlin im Kaiserreich, Berlin 1999 (Interdisziplinäre Arbeitsgruppen, Berlin-Brandenburgische Akademie der Wissenschaften: Forschungsberichte, 7).

Marchand, Suzanne L., Down from Olympus. Archaeology and Philhellenism in Germany, 1750-1970, Princeton 1996.

Momigliano, Arnaldo, »Epilogo senza conclusione«, in: Les études classiques aux XIXe et XXe siècles. Leur place dans l'histoire des idées, hg. v. Willem den Boer, Genèvel Vandoeuvres 1979 (Entretiens sur l'Antiquité Classique, 26), 305-317.

Mommsen, Theodor, »Antrittsrede als Mitglied der Akademie«, in: Monatsberichte der Berliner Akademie (1858), 393-395 [Nachdruck in: ders., Reden und Aufsätze, hg. v. Otto Hirschfeld, Berlin 1905, 35-38].

Mommsen, Theodor, "Rede gehalten am 2. Juli in der öffentlichen Sitzung der Akademie zur Feier des Leibniz'schen Jahrestages«, in: Monatsberichte der Berliner Akademie (1874), 449-458 [Nachdruck in: ders., Reden und Aufsätze, hg. v. Otto Hirschfeld, Berlin 1905, 39-49].

Mommsen, Theodor, »Antwort auf die Antwortrede von Adolf Harnack [1890]《, in: ders., Reden und Aufsätze, hg. v. Otto Hirschfeld, Berlin 1905, 208-210.

Mommsen, Theodor, »Ansprache am Leibniz'schen Gedächtnistage am 4. Juli 1895«, in: Sitzungsberichte der Preußischen Akademie der Wissenschaften, Philosophischhistorische Klasse (1895), 733-735 [Nachdruck in: ders., Reden und Aufsätze, hg. v. Otto Hirschfeld, Berlin 1905, 196-198]. 
Mommsen, Theodor, Reden und Aufsätze, hg. v. Otto Hirschfeld, Berlin 1905 [3. Aufl. 1912].

Nowak, Kurt, »Wissenschaft und Weltgestaltung auf dem Boden des modernen Protestantismus«, in: Adolf von Harnack als Zeitgenosse. Reden und Schriften aus den Jahren des Kaiserreichs und der Weimarer Republik, hg. v. Kurt Nowak, Bd. 1, Berlin/New York 1996, 1-99.

Rebenich, Stefan, Theodor Mommsen und Adolf Harnack. Wissenschaft und Politik im Berlin des ausgehenden 19. Jahrhunderts. Mit einem Anhang: Edition und Kommentierung des Briefwechsels, Berlin/New York 1997.

Rebenich, Stefan, »Die Altertumswissenschaften und die Kirchenväterkormmission an der Akademie: Theodor Mommsen und Adolf Harnack«, in: Die Königlich Preußische Akademie der Wissenschaften zu Berlin im Kaiserreich, hg. v. Jürgen Kocka, Berlin 1999a (Interdisziplinäre Arbeitsgruppen, Berlin-Brandenburgische Akademie der Wissenschaften: Forschungsberichte, 7), 199-233.

Rebenich, Stefan, »>Mommsen ist er niemals näher getreten<. Theodor Mommsen und Hermann Diels", in: Hermann Diels (1848-1922) et la science de l'antiquité, hg. v. William M. Calder III/Jaap Mansfeld, Genève/Vandoeuvres 1999b (Entretiens sur l'Antiquité Classique, 45), 85-142.

Rebenich, Stefan, Artikel »Historismus I. Allgemein«, in: Der Neue Pauly 14 (2000), $469-485$.

Rebenich, Stefan, "Zwischen Anpassung und Widerstand? Die Berliner Akademie der Wissenschaften von 1933 bis 1945«, in: Antike und Altertumswissenschaft in der Zeit von Faschismus und Nationalsozialismus. Kolloquium Universität Zürich (14.17. Oktober 1998), hg. v. Beat Näf, Mandelbachtal/Cambridge 2001a (Texts and Studies in the History of Humanities, 1), 203-244.

Rebenich, Stefan, $\gg$ Der alte Meergreis, die Rose von Jericho und ein höchst vortrefflicher Schwiegersohn: Mommsen, Harnack und Wilamowitz«, in: Adolf von Harnack. Theologe, Historiker, Wissenschaftspolitiker, hg. v. Kurt Nowak/Otto G. Oexle, Göttingen $2001 \mathrm{~b}$ (Veröffentlichungen des Max-Planck-Instituts für Geschichte, 161), 39-69.

Rebenich, Stefan, »Alte Geschichte zwischen Demokratie und Diktatur. Der Fall Helmut Berve«, in: Chiron 31 (2001c), 457-496.

Rebenich, Stefan, Theodor Mommsen. Eine Biographie, München 2002.

Rebenich, Stefan, »Adolf Erman und die Berliner Akademie der Wissenschaften «, in: $\ddot{A}$ gyptologie als Wissenschaft. Adolf Erman (1854-1937) in seiner Zeit, hg. v. Bernd U. Schipper, Berlin/New York 2006, 340-370.

Rebenich, Stefan, »>Dass ein strahl von Hellas auf euch fiel - Platon im Georgekreis«, in: George-Jahrbuch 7 (2008/2009), 115-141.

Sachse, Arnold, Friedrich Althoff und sein Werk, Berlin 1928.

Schiera, Pierangelo, Laboratorium der bürgerlichen Welt. Deutsche Wissenschaft im 19. Jahrhundert, Frankfurt am Main 1992.

Stähli, Adrian, »Vom Ende der Klassischen Archäologie«, in: Posthumanistische Klassische Archäologie. Historizität und Wissenschaftlichkeit von Interessen und Methoden. Internationales Kolloquium (Berlin, 19,-21. Februar 1999), hg. v. Stefan Altekamp/ Mathias Hofter/Michael Krumme, München 2001, 145-170, 475-507.

Timpe, Dieter, $\gg$ Die Alte Geschichte und das moderne Geschichtsbewußtsein «, in: Geschichte in Wissenschaft und Unterricht 24 (1973), 645-658 [Nachdruck in: Wilfried Nippel (Hg.), Über das Studium der Alten Geschichte, München 1993, 353372]. 
Tommissen, Piet, Schmittiana, Bd. 1, 3. Aufl. Brüssel 1990.

Unte, Wolfhart, »Wilamowitz als wissenschaftlicher Organisator«, in: Wilamowitz nach 50 Jahren. Symposium aus Anlaß des 50. Todestages, hg. v. William M. Calder III/Hellmut Flashar/Theodor Lindken, Darmstadt 1985, 720-770 [Nachdruck in: ders., Heroen und Epigonen. Gelehrtenbiographien der klassischen Altertumswissenschaft im 19. und 20. Jahrhundert, St. Katharinen 2003, 271-329].

Unte, Wolfhart, Heroen und Epigonen. Gelehrtenbiographien der klassischen Altertumswissenschaft im 19. und 20. Jahrhundert, St. Katharinen 2003.

vom Brocke, Bernhard, »Hochschul- und Wissenschaftspolitik in Preußen und im Deutschen Kaiserreich 1882-1907: Das >System Althoff< «, in: Bildungspolitik in Preußen zur Zeit des Kaiserreichs, hg. v. Peter Baumgart, Stuttgart 1980 (Preußen in der Geschichte, 1), 9-118.

vom Brocke, Bernhard (Hg.), Wissenschaftsgeschichte und Wissenschaftspolitik im Industriezeitalter. Das »System Althoff " in historischer Perspektive, Hildesheim 1991 (Geschichte von Bildung und Wissenschaft, Reihe B, Sammelwerke, 5).

Vom Bruch, Rüdiger, »Mommsen und Harnack: Die Geburt von Big Science aus den Geisteswissenschaften«, in: Theodor Mommsen. Wissenschaft und Politik im 19. Jahrhundert, hg. v. Alexander Demandt/Andreas Goltz/Heinrich SchlangeSchöningen, Berlin/New York 2005, 121-141.

Walther, Peter Th., »Honoratiorenklub oder Forschungsstätte. Die Statutendebatte der Akademie 1874 bis 1881«, in: Die Königlich Preußische Akademie der Wissenschaften zu Berlin im Kaiserreich, hg. v. Jürgen Kocka, Berlin 1999 (Interdisziplinäre Arbeitsgruppen, Berlin-Brandenburgische Akademie der Wissenschaften: Forschungsberichte, 7), 103-118.

Wilamowitz-Moellendorff, Ulrich von, Reden und Vorträge, Berlin 1901.

Wilamowitz-Moellendorff, Ulrich von, "Geschichte der Philologie«, in: Einleitung in die Altertumswissenschaft, hg. v. Alfred Gercke/Eduard Norden, Bd. 1, Heft 1, 3. Aufl. Leipzig/Berlin 1927.

Wilamowitz-Moellendorff, Ulrich von, Erinnerungen 1848-1914, 2. Aufl. Leipzig 1929.

Wilamowitz-Moellendorff, Ulrich von, »Der griechische Unterricht auf dem Gymnasium [1901]«, in: ders., Kleine Schriften, Bd. VI: Philologiegeschichte, Pädagogik und Verschiedenes, Nachlese zu den Bänden I und II, Nachtrag zur Bibliographie, Berlin $1972,77-89$.

Wirtz, Thomas, »Der Pendler Carl Schmitt. Zwischen Preußen und dem Sauerland «, in: Preußische Stile. Ein Staat als Kunststück, hg. v. Patrick Bahners/Gerd Roellecke, Stuttgart 2001, 406-415.

\section{Abbildungsnachweis}

Abb. 1: Akademiebibliothek, Berlin-Brandenburgische Akademie der Wissenschaften.

Abb. 2: Photo (C) Archiv zur Geschichte der Max-Planck-Gesellschaft, vgl. Sachse, Arnold, Friedrich Althoff und sein Werk, Berlin 1928, 1 (nach dem Titelblatt).

Abb. 3: Gemälde von Wilhelm Pape, in: Grau, Conrad, Berühmte Wissenschaftsakademien. Von ihrem Entstehen und ihrem weltweiten Erfolg, Leipzig 1988, 220. 